\title{
LA CONTRIBUCIÓN DE LA CIENCIA A LA CULTURA CIUDADANA
}

Publicado en la revista: Cultura y Educación, 16 (3) 259-272

Daniel Gil y Amparo Vilches.

Universitat de València

Correspondencia: Departamento de Didáctica de las Ciencias Experimentales y Sociales. Universitat de València. Apartado de Correos 22045, 46071 Valencia.

Datos sobre los autores: Daniel Gil, profesor de Didáctica de las Ciencias de la Universidad de Valencia y Amparo Vilches, profesora del IES Sorolla de Valencia, han investigado en torno a los distintos aspectos del proceso de enseñanza aprendizaje de las ciencias, centrándose en la actualidad en el de la formación del profesorado, la alfabetización científica y las relaciones Ciencia, Tecnología, Sociedad y Ambiente.

Resumen: El propósito de este trabajo es analizar las contribuciones de la ciencia a la cultura ciudadana, con objeto de fundamentar las propuestas de alfabetización científica como una componente esencial de la educación, susceptible de favorecer la participación ciudadana en la toma de decisiones acerca de los problemas relacionados con el desarrollo tecnocientífico, contribuir a la formación de un espíritu crítico y transmitir la emoción de los apasionantes desafíos a los que se ha enfrentado la comunidad científica. Se pretende responder así a las críticas de algunos autores, que se han referido a la alfabetización científica del conjunto de ciudadanos y ciudadanas como un mito irrealizable.

Descriptores: Alfabetización científica; inmersión en una cultura científica y tecnológica; relaciones ciencia-tecnología-sociedad-ambiente (CTSA); toma de decisiones; principio de prudencia; movimientos anti-ciencia.

\section{CONTRIBUTION OF SCIENCE TO CITIZENS' CULTURE}

\begin{abstract}
Our aim in this paper is to analyse what science can bring to citizens' culture, in order to justify the proposals of scientific literacy as an essential component of education. We intend to show that scientific literacy is necessary to:

- favour citizens' participation in decision making about problems related to scientific and technological development

- contribute to the development of critical thinking

- transmit the emotion of the exciting challenges faced by the scientific community throughout history and nowadays.

We shall try, in this way, to respond to the criticisms of some authors, who have referred to scientific literacy of citizens as an unattainable myth.
\end{abstract}

Key words: Scientific literacy; Immersion in a scientific and technological culture; Science-Technology-Environment-Society relationships; Decision making; Precaution principle; Anti-science social movements. 


\section{LA CONTRIBUCIÓN DE LA CIENCIA A LA CULTURA CIUDADANA}

Daniel Gil y Amparo Vilches.

Universitat de València

\section{LA ALFABETIZACIÓN CIENTÍFICA, ¿NECESIDAD OBVIA O MITO IRREALIZABLE?}

Parece existir un amplio consenso en contemplar la alfabetización científica como una prioridad de la educación, un factor esencial del desarrollo de las personas y de los pueblos. Así, en la Conferencia Mundial sobre la Ciencia para el siglo XXI, auspiciada por la UNESCO y el Consejo Internacional para la Ciencia, se declara: "Para que un país esté en condiciones de atender a las necesidades fundamentales de su población, la enseñanza de las ciencias y la tecnología es un imperativo estratégico (...) Hoy más que nunca es necesario fomentar y difundir la alfabetización científica en todas las culturas y en todos los sectores de la sociedad, (...) a fin de mejorar la participación de los ciudadanos en la adopción de decisiones relativas a la aplicaciones de los nuevos conocimientos" (Declaración de Budapest, 1999).

Por poner otro ejemplo relevante, podemos mencionar los National Science Education Standards, auspiciados por el National Research Council (1996) para el logro de la educación científica de los ciudadanos y ciudadanas estadounidenses del siglo XXI, en cuya primera página podemos leer: "En un mundo repleto de productos de la indagación científica, la alfabetización científica se ha convertido en una necesidad para todos: todos necesitamos utilizar la información científica para realizar opciones que se plantean cada día; todos necesitamos ser capaces de implicarnos en discusiones públicas acerca de asuntos importantes que se relacionan con la ciencia y la tecnología; y todos merecemos compartir la emoción y la realización personal que puede producir la comprensión del mundo natural”.

La importancia concedida a una educación científica para todos es tal, que ha llevado a establecer una analogía entre la alfabetización básica iniciada el siglo pasado y el actual movimiento de alfabetización científica y tecnológica (Fourez, 1997).

Podría parecer innecesario, pues, argumentar la necesidad de incorporar la educación científica a la cultura general. Sin embargo, algunos autores han venido a poner en duda la conveniencia e incluso la posibilidad de que la generalidad de los ciudadanos y ciudadanas adquieran una formación científica realmente útil (Atkin y Helms, 1993 ; Shamos, 1995; Fensham 2002 a 2002 ). Se trata de artículos bien documentados que pretenden "sacudir aparentes evidencias", como sería, en su opinión, la necesidad de alfabetizar científicamente a 
toda la población, algo que Shamos califica de auténtico mito en su libro The Myth Of Scientific Literacy (Shamos, 1995). Conviene, pues, prestar atención a los argumentos críticos de estos autores y analizar más cuidadosamente las razones que justifican las propuestas de "ciencia para todos".

En opinión de Fensham (2002 b), el movimiento ciencia para todos y las primeras discusiones sobre la alfabetización científica se basaban en dos ideas preconcebidas. La primera, que denomina tesis pragmática, considera que, dado que las sociedades se ven cada vez más influidas por las ideas y productos de la ciencia y, sobre todo, de la tecnología, los futuros ciudadanos se desenvolverán mejor si adquieren una base de conocimientos científicos. La segunda, o tesis democrática, supone que la alfabetización científica permite a los ciudadanos participar en las decisiones que las sociedades deben adoptar en torno a problemas sociocientíficos y socio-tecnológicos cada vez más complejos.

Pero la tesis pragmática, afirma Fensham, no tiene en cuenta el hecho de que la mayoría de los productos tecnológicos están concebidos para que los usuarios no tengan ninguna necesidad de conocer los principios científicos en los que se basan para poder utilizarlos. Hay que reconocer que ésta es una crítica fundamentada: nadie puede desenvolverse hoy sin saber leer y escribir o sin dominar las operaciones matemáticas más simples, pero millones de ciudadanos, incluidas eminentes personalidades, en cualquier sociedad, reconocen su falta de conocimientos científicos, sin que ello haya limitado para nada su vida práctica. La analogía entre alfabetización básica y alfabetización científica, concluyen por ello Atkin y Helms (1993), no se sostiene.

Por lo que respecta a la tesis democrática, pensar que una sociedad científicamente alfabetizada está en mejor situación para actuar racionalmente frente a los problemas sociocientíficos, constituye, según Fensham, una ilusión que ignora la complejidad de los conceptos científicos implicados, como sucede, por ejemplo, en el calentamiento global. Es absolutamente irrealista, añade, creer que este nivel de conocimientos pueda ser adquirido, ni siquiera en las mejores escuelas. Un hecho clarificador a ese respecto es el resultado del Project 2061, financiado por la American Association for the Advancement of Sciences (AAAS), que consistió en pedir a un centenar de eminentes científicos de distintas disciplinas que enumeraran los conocimientos científicos que deberían impartirse en los años de escolarización obligatoria para garantizar una adecuada alfabetización científica de los niños y niñas norteamericanos. El número total de aspectos a cubrir, señala Fensham, desafía el 
entendimiento y resulta superior a la suma de todos los conocimientos actualmente enseñados a los estudiantes de élite que se preparan como futuros científicos.

Argumentos como éstos son los que llevan a autores como Shamos, Fensham, etc., a considerar la alfabetización científica como un mito irrealizable, causante, además, de un despilfarro de recursos. ¿Debemos, pues, renunciar, a la idea de una educación científica básica para todos? En cualquier caso, críticas como las de Fensham obligan, a quienes concebimos la alfabetización científica como una componente esencial de las humanidades, a profundizar en las razones que recomiendan que la educación científica y tecnológica forme parte de una cultura general para toda la ciudadanía, sin darlo simplemente por sentado como algo obvio.

Se trata, en definitiva, de considerar con cierta atención, qué puede realmente aportar la educación científica y tecnológica a la formación ciudadana. Esto es lo que nos proponemos hacer en este trabajo, estudiando, como se indica en el título, cuál puede ser la contribución de la educación científica a la cultura ciudadana. Procederemos, para ello, a discutir sus posibles contribuciones en:

- la toma de decisiones acerca de los problemas a los que se enfrenta la humanidad

- la formación de un espíritu crítico

- el disfrute personal.

CONTRIBUCIÓN DE LA FORMACIÓN CIENTÍFICA $Y$ TECNOLÓGICA A LA TOMA DE DECISIONES SOBRE LOS PROBLEMAS QUE AFECTAN A LA HUMANIDAD

Como hemos señalado, tanto la Conferencia Mundial sobre la Ciencia para el siglo XXI (Declaración de Budapest, 1999) como los National Science Education Standards (National Research Council, 1996) ponen el acento en la necesidad de una formación científica que permita a la ciudadanía participar en la toma de decisiones, en asuntos que se relacionan con la ciencia y la tecnología.

Este argumento "democrático" es, quizás, el más ampliamente utilizado por quienes reclaman la alfabetización científica y tecnológica como una componente básica de la educación ciudadana (Fourez, 1997; Bybee, 1997; DeBoer, 2000; Marco 2000...). Y es también el que autores como Fensham (2002 a y 2002 b) cuestionan más directa y explícitamente, argumentando, como hemos visto, que el conocimiento científico, susceptible de orientar la toma de decisiones, exige una profundización que sólo es accesible a los especialistas. Analizaremos, pues, sus argumentos, que no son, en absoluto, triviales, y que, en su opinión y 
en la de otros autores en quienes se apoyan, cuestionarían las propuestas de educación científica para todos.

Previamente, sin embargo, nos referiremos a una segunda corriente de pensamiento que cuestiona aún más profundamente el supuesto papel democratizador de la formación científica, pues atribuye al propio desarrollo tecnocientífico la responsabilidad de los problemas a los que la humanidad ha de hacer frente hoy, desde las diversas formas de contaminación ambiental a las armas de destrucción masiva, que dibujan un negro panorama de emergencia planetaria. Ello está dando lugar a un creciente movimiento anticiencia, que afecta a un buen número de estudiantes. Analizaremos seguidamente estas dos corrientes críticas que, con argumentos diferentes, vienen ambas a cuestionar la necesidad de una alfabetización científica para favorecer la participación ciudadana en la toma de decisiones.

\section{¿El desarrollo tecnocientífico como origen de los problemas?}

La crítica al papel de la ciencia y la tecnología en el deterioro de nuestras condiciones de vida parece contar con serios argumentos: ¿No son acaso científicos los creadores de los Contaminantes Orgánicos Persistentes, como el DDT y tantos otros, que están envenenando el planeta? ¿No lo eran quienes pusieron a punto la tecnología nuclear que nos ha legado esa bomba de relojería que suponen los residuos radiactivos o quienes han creado los freones responsables de la destrucción de la capa de ozono? ¿No es un producto tecnológico ese desarrollo industrial que incrementa el efecto invernadero y amenaza con un cambio climático destructor de los frágiles equilibrios que hacen posible nuestra vida?

Sin embargo, una respuesta adecuada a estas cuestiones debe tener en cuenta que hoy la ciencia y la tecnología lo impregnan todo y es casi imposible encontrar algo, sea bueno o malo, en lo que no estén jugando un papel. La lista de contribuciones de la tecnociencia al bienestar humano sería al menos igualmente larga que la de sus efectos negativos. Además, como señala el historiador de la ciencia Sánchez Ron (1994), “es el conocimiento científico quien nos hace ser conscientes de algunos problemas medioambientales. ¿Conoceríamos sin la ciencia que existen agujeros en la capa de ozono? Y en lo que se refiere a identificar con claridad cuáles son las causas de prácticamente todo el deterioro de la naturaleza, ¿existe mejor analista que el científico?". No podemos ignorar, efectivamente, que son científicos quienes estudian los problemas a los que se enfrenta hoy la humanidad, advierten de los riesgos y ponen a punto soluciones. Por supuesto no sólo científicos, ni todos los científicos. Por otra parte, es cierto que han sido científicos los productores de, por ejemplo, los freones 
que destruyen la capa de ozono. Pero, no lo olvidemos, junto a empresarios, economistas, trabajadores, políticos... La tendencia a descargar sobre la ciencia y la tecnología la responsabilidad de la situación actual de deterioro creciente, no deja de ser una nueva simplificación maniquea en la que resulta fácil caer. Las críticas y las llamadas a la responsabilidad han de extenderse a todos nosotros, incluidos los "simples" consumidores de los productos nocivos (Vilches y Gil, 2003). Y ello supone hacer partícipe a la ciudadanía de la responsabilidad de la toma de decisiones en torno a este desarrollo tecnocientífico. Pero esto choca con los argumentos de Fensham en contra de la posibilidad de una alfabetización científica realmente efectiva para hacer posible esa participación ciudadana. Argumentos que merecen un detenido análisis, puesto que si resultara cierta la supuesta inaccesibilidad de los conocimientos científicos necesarios para proceder a la toma de decisiones fundamentadas, no sólo perderían sentido las propuestas de alfabetización científica, sino la idea misma de participación ciudadana en la toma de decisiones.

\section{¿Permite la alfabetización científica la participación en la toma de decisiones?}

Intentaremos mostrar, sin embargo, que esa participación, en la toma fundamentada de decisiones, precisa de los ciudadanos más que un nivel de conocimientos muy elevado, la vinculación de un mínimo de conocimientos específicos, perfectamente accesible a la ciudadanía, con planteamientos globales y consideraciones éticas que no exigen especialización alguna. Más concretamente, intentaremos mostrar que la posesión de profundos conocimientos específicos, como los que poseen los especialistas en un campo determinado, no garantiza la adopción de decisiones adecuadas, sino que se necesitan enfoques que contemplen los problemas en una perspectiva más amplia, analizando las posibles repercusiones a medio y largo plazo, tanto en el campo considerado como en otros. Y eso es algo a lo que pueden contribuir no especialistas, con perspectivas e intereses más amplios, siempre que posean un mínimo de conocimientos científicos específicos sobre la problemática estudiada, sin los cuales resulta imposible comprender las opciones en juego y participar en la adopción de decisiones fundamentadas. Esperamos responder, de este modo, a los argumentos de quienes consideran la alfabetización científica del conjunto de la ciudadanía un mito irrealizable y sin verdadero interés.

Analizaremos para ello, como ejemplo paradigmático, el problema creado por los fertilizantes químicos y pesticidas que, a partir de la Segunda Guerra Mundial, produjeron una verdadera revolución agrícola, incrementando notablemente la producción. Recordemos que la 
utilización de productos de síntesis para combatir los insectos, plagas, malezas y hongos aumentó la productividad en un periodo en el que un notable crecimiento de la población mundial lo exigía. Y recordemos igualmente que algunos años después la Comisión Mundial del Medio Ambiente y del Desarrollo (1988) advertía que su exceso constituye una amenaza para la salud humana, provocando desde malformaciones congénitas hasta cáncer, y siendo auténticos venenos para peces, mamíferos y pájaros. Por ello dichas sustancias, que se acumulan en los tejidos de los seres vivos, han llegado a ser denominadas, junto con otras igualmente tóxicas, "Contaminantes Orgánicos Persistentes" (COP).

Este envenenamiento del planeta por los productos químicos de síntesis, y en particular por el DDT, ya había sido denunciado a finales de los años 50 por Rachel Carson (1980) en su libro Primavera silenciosa (título que hace referencia a la desaparición de los pájaros) en el que daba abundantes y contrastadas pruebas de los efectos nocivos del DDT... lo que no impidió que fuera violentamente criticada y sufriera un acoso muy duro por parte de la industria química, los políticos y numerosos científicos, que negaron valor a sus pruebas y le acusaron de estar contra un progreso que permitía dar de comer a una población creciente y salvar así muchas vidas humanas. Sin embargo, apenas 10 años más tarde se reconoció que el DDT era realmente un peligroso veneno y se prohibió su utilización en el mundo rico, aunque, desgraciadamente, se siguió utilizando en los países en desarrollo.

Lo que nos interesa destacar aquí es que la batalla contra el DDT fue dada por científicos como Rachel Carson en confluencia con grupos ciudadanos que fueron sensibles a sus llamadas de atención y argumentos. De hecho Rachel Carson es hoy recordada como "madre del movimiento ecologista", por la enorme influencia que tuvo su libro en el surgimiento de grupos activistas que reivindicaban la necesidad de la protección del medio ambiente, así como en los orígenes del denominado movimiento CTS. Sin la acción de estos grupos de ciudadanos y ciudadanas con capacidad para comprender los argumentos de Carson, la prohibición se hubiera producido mucho más tarde, con efectos aún más devastadores. Conviene llamar la atención sobre la influencia de estos "activistas ilustrados" y su indudable participación en la toma de decisiones, al hacer suyos los argumentos de Carson y exigir controles rigurosos de los efectos del DDT, que acabaron convenciendo a la comunidad científica y, posteriormente, a los legisladores, obligando a su prohibición. Y conviene señalar también que muchos científicos, con un nivel de conocimientos sin duda muy superior al de esos ciudadanos, no supieron o quisieron ver inicialmente los peligros asociados al uso de plaguicidas. 
Podemos mencionar muchos otros ejemplos similares, como, entre otros, los relacionados con la construcción de las centrales nucleares y el almacenamiento de los residuos radiactivos; el uso de los "freones" (compuestos fluorclorocarbonados), destructores de la capa de ozono; el incremento del efecto invernadero, debido fundamentalmente a la creciente emisión de $\mathrm{CO}_{2}$, que amenaza con un cambio climático global de consecuencias devastadoras; los alimentos manipulados genéticamente, etc., etc.

Conviene detenerse mínimamente en el ejemplo de los alimentos transgénicos, que está suscitando hoy los debates más encendidos y que puede ilustrar perfectamente el papel de la ciudadanía en la toma de decisiones. También en este terreno las cosas empezaron planteándose como algo positivo que, entre otras ventajas, podría reducir el uso de pesticidas y herbicidas y convertirse en "la solución definitiva para los problemas del hambre en el mundo". Algo que, además, abría enormes posibilidades en el campo de la salud, para el tratamiento o curación de enfermedades incurables con los conocimientos y técnicas actuales. Así, en 1998, el director general de una de las más fuertes y conocidas empresas de organismos manipulados genéticamente (OGM) y alimentos derivados, en la asamblea anual de la Organización de la Industria de la Biotecnología, afirmó que, "de algún modo, vamos a tener que resolver cómo abastecer de alimentos a una demanda que duplica la actual, sabiendo que es imposible doblar la superficie cultivable. Y es imposible, igualmente, aumentar la productividad usando las tecnologías actuales, sin crear graves problemas a la sostenibilidad de la agricultura (...) La biotecnología representa una solución potencialmente sostenible al problema de la alimentación”.

Pero no todos han estado de acuerdo con una visión tan optimista y muy pronto surgieron las preocupaciones por sus posibles riesgos para el medio ambiente, para la salud humana, para el futuro de la agricultura, etc. Una vez más, señalaron los críticos, se pretende proceder a una aplicación apresurada de tecnologías cuyas repercusiones no han sido suficientemente investigadas, sin tener garantías razonables de que no aparecerán efectos nocivos... como ocurrió con los plaguicidas, que también fueron saludados como "la solución definitiva" al problema del hambre y de muchas enfermedades infecciosas.

Nos encontramos, pues, con un amplio debate abierto, con estudios inacabados y resultados parciales contrapuestos (muchos de ellos presentados por las propias empresas productoras). Esas discrepancias entre los propios científicos son esgrimidas en ocasiones como argumento para cuestionar la participación de la ciudadanía en un debate "en el que ni siquiera los científicos, con conocimientos muy superiores, se ponen de acuerdo". Pero cabe insistir, una 
vez más, en que la toma de decisiones no puede basarse exclusivamente en argumentos científicos específicos. Por el contrario, las preocupaciones que despierta la utilización de estos productos y las dudas a cerca de sus repercusiones recomiendan que los ciudadanos y ciudadanas tengan la oportunidad de participar en el debate y exigir una estricta aplicación del principio de prudencia. Ello no cuestiona, desde luego, el desarrollo de la investigación ni en éste ni en ningún otro campo, pero se opone a la aplicación apresurada, sin suficientes garantías, de los nuevos productos, por el afán del beneficio a corto plazo. Es absolutamente lógico, pues, que haya surgido un amplio movimiento de rechazo entre los consumidores, apoyado por un amplio sector de la comunidad científica, hacia la comercialización precipitada y poco transparente de estos alimentos manipulados genéticamente. Cabe señalar que este rechazo está dando notables frutos, como la firma en Montreal del Protocolo de Bioseguridad en febrero de 2000 por 130 países, a pesar de las enormes dificultades previas y presiones de los países productores de organismos modificados genéticamente. Dicho protocolo, enmarcado en el Convenio sobre Seguridad Biológica de la ONU, supone un paso importante en la legislación internacional (aunque todavía no plenamente consolidado, por la falta de firmas como la de EEUU), puesto que obliga a demostrar la seguridad antes de comercializar los productos, evitando así que se repitan los graves errores del pasado.

Debemos insistir en que esta participación de la ciudadanía en la toma de decisiones, que se traduce, en general, en evitar la aplicación apresurada de innovaciones de las que se desconocen las consecuencias a medio y largo plazo, no supone ninguna rémora para el desarrollo de la investigación, ni para la introducción de innovaciones para las que existan razonable garantías de seguridad. De hecho la opinión pública no se opone, por ejemplo, a la investigación con células madre embrionarias. Muy al contrario, está apoyando a la mayoría de la comunidad científica que reclama se levante la prohibición introducida en algunos países, debido a la presión de grupos ideológicos fundamentalistas.

En definitiva, la participación ciudadana en la toma de decisiones es hoy un hecho positivo, una garantía de aplicación del principio de precaución, que se apoya en una creciente sensibilidad social frente a las implicaciones del desarrollo tecnocientífico que puedan comportar riesgos para las personas o el medio ambiente. Dicha participación, hemos de insistir, reclama un mínimo de formación científica que haga posible la comprensión de los problemas y de las opciones - que se pueden y se deben expresar con un lenguaje accesible- y no ha de verse rechazada con el argumento de que problemas como el cambio climático o la manipulación genética sean de una gran complejidad. Naturalmente se precisan estudios 
científicos rigurosos, pero tampoco ellos, por si solos, bastan para adoptar decisiones adecuadas, puesto que, a menudo, la dificultad estriba, antes que en la falta de conocimientos, en la ausencia de un planteamiento global que evalúe los riesgos y contemple las posibles consecuencias a medio y largo plazo. Muy ilustrativo a este respecto puede ser el enfoque dado a las catástrofes anunciadas, como la provocada por el hundimiento del Prestige y otros petroleros, que se intenta presentar como "accidentes" (Vilches y Gil, 2003).

Todo ello constituye un argumento decisivo a favor de una alfabetización científica del conjunto de la ciudadanía, cuya necesidad aparece cada vez con más claridad ante la situación de auténtica "emergencia planetaria" (Bybee, 1991) que estamos viviendo. Así, en la Conferencia de las Naciones Unidas sobre Medio Ambiente y Desarrollo, celebrada en Río de Janeiro en 1992 y conocida como Primera Cumbre de la Tierra, se reclamó una decidida acción de los educadores para que los ciudadanos y ciudadanas adquieran una correcta percepción de cuál es esa situación y puedan participar en la toma de decisiones fundamentadas (Edwards et al., 2001; Gil et al., 2003). Como señalan Hicks y Holden (1995), si los estudiantes han de llegar a ser ciudadanos y ciudadanas responsables, es preciso que les proporcionemos ocasiones para analizar los problemas globales que caracterizan esa situación de emergencia planetaria y considerar las posibles soluciones.

Así pues, la alfabetización científica no sólo no constituye un "mito irrealizable" (Shamos, 1995), sino que se impone como una dimensión esencial de la cultura ciudadana. Cabe señalar, por otra parte, que la reivindicación de esta dimensión no es el fruto de "una idea preconcebida" aceptada acríticamente, como afirma Fensham (2002 a y 2002 ). Muy al contrario, el prejuicio ha sido y sigue siendo que "la mayoría de la población es incapaz de acceder a los conocimientos científicos, que exigen un alto nivel cognitivo", lo que implica, obviamente, reservarlos a una pequeña élite. El rechazo de la alfabetización científica recuerda así la sistemática resistencia histórica de los privilegiados a la extensión de la cultura y a la generalización de la educación (Gil y Vilches, 2001). Y su reivindicación forma parte de la batalla de las fuerzas progresistas por vencer dichas resistencias, que constituyen el verdadero prejuicio acrítico. Podemos recordar a este respecto la frase del gran científico francés Paul Langevin, quien en 1926 escribía: "En reconocimiento del papel jugado por la ciencia en la liberación de los espíritus y la confirmación de los derechos del hombre, el movimiento revolucionario hace un esfuerzo considerable para introducir la enseñanza de las ciencias en la cultura general y conformar esas humanidades modernas que aún no hemos logrado establecer". Ello nos remite a lo que, para muchos, puede constituir la principal 
aportación de la ciencia a la cultura ciudadana: su contribución al desarrollo del espíritu crítico, que abordaremos en el siguiente apartado.

\section{CONTRIBUCIÓN DE LA CIENCIA A LA FORMACIÓN DEL ESPÍRITU CRÍTICO}

Las palabras de Langevin que acabamos de recordar expresan el reconocimiento "del papel jugado por la ciencia en la liberación de los espíritus", es decir, de su contribución, a lo largo de la historia, a la formación de un espíritu crítico capaz de cuestionar dogmas y desafiar autoritarismos y privilegios.

Frente a esta valoración, sin embargo, existe hoy en día un creciente movimiento "anticiencia", que denuncia la "ciencia exacta" como un nuevo dogmatismo de conocimientos esclerotizados y, a menudo, carentes de interés. "¿Qué interés formativo puede tener para los jóvenes -se pregunta- el estudio de materias abstractas y puramente formales como la Mecánica?".

La crítica puede considerarse justa si se refiere a la forma en que la enseñanza presenta habitualmente esas materias. Pero, ¿cómo aceptar que el desarrollo de la Mecánica, o de cualquier otro campo de la ciencia, constituya una materia abstracta, puramente formal? Basta asomarse a la historia de las ciencias para darse cuenta del carácter de verdadera aventura, de lucha apasionada y apasionante por la libertad de pensamiento -en la que no han faltado ni persecuciones ni condenas- que el desarrollo científico ha tenido.

La recuperación de esos aspectos históricos y de relación ciencia-tecnología-sociedadambiente (CTSA), sin dejar de lado los problemas que han jugado un papel central en el cuestionamiento de dogmatismos y en la defensa de la libertad de investigación y pensamiento, puede contribuir a devolver al aprendizaje de las ciencias la vitalidad y relevancia del propio desarrollo científico. Los debates en torno al heliocentrismo, el evolucionismo, la síntesis orgánica, el origen de la vida... constituyen ejemplos relevantes.

Pero el aprendizaje de las ciencias puede y debe ser también una aventura potenciadora del espíritu crítico en un sentido más profundo: la aventura que supone enfrentarse a problemas abiertos, participar en la construcción tentativa de soluciones... la aventura, en definitiva, de hacer ciencia. El problema es que la naturaleza de la actividad científica aparece distorsionada en la educación científica, incluso universitaria. Ello plantea la necesidad de superación de visiones deformadas y empobrecidas de la ciencia y la tecnología, socialmente aceptadas, que afectan al propio profesorado, para proporcionar una visión más creativa, abierta y socialmente contextualizada, acorde con la propia naturaleza tentativa de la actividad 
científica (Fernández et al., 2002), en la que el espíritu crítico, cuestionador de las apariencias, juega un papel esencial.

Cabe señalar que una "justificación" del reduccionismo operativista habitual de la educación científica estriba, paradójicamente, en "las exigencias de la formación de futuros científicos" (Gil y Vilches, 2001). Nos detendremos en analizar los argumentos utilizados.

\section{¿Se contrapone la alfabetización científica de la ciudadanía a la preparación de los futuros científicos?}

Una tesis comúnmente aceptada por los diseñadores de currículos y los profesores de ciencias es que la educación científica ha estado orientada hasta aquí para preparar a los estudiantes como si todos pretendieran llegar a ser especialistas en biología, física o química. Por ello -se afirma- los currículos planteaban, como objetivos prioritarios, que los estudiantes supieran, fundamentalmente, los conceptos, principios y leyes de esas disciplinas.

Dicha orientación habría de modificarse -se explica- a causa de que la educación científica se plantea ahora como parte de una educación general para todos los futuros ciudadanos y ciudadanas. Ello es lo que justifica, se afirma, el énfasis de las nuevas propuestas curriculares en los aspectos sociales y personales, puesto que se trata de ayudar a la gran mayoría de la población a tomar conciencia de las complejas relaciones ciencia y sociedad, para permitirles participar en la toma de decisiones y, en definitiva, a considerar la ciencia como parte de la cultura de nuestro tiempo.

Esta apuesta por una educación científica orientada a la formación ciudadana, en vez de a la preparación de futuros científicos, genera resistencias en numerosos profesores, quienes argumentan, legítimamente, que la sociedad necesita científicos y tecnólogos que han de formarse y ser adecuadamente seleccionados desde los primeros estadios.

En ambas actitudes -tanto la que defiende la alfabetización científica para todos, como la que prioriza la formación de futuros científicos- se aprecia claramente una misma aceptación de la contraposición entre dichos objetivos. Pero es preciso denunciar la falacia de esta contraposición entre ambas orientaciones curriculares y de los argumentos que supuestamente la avalan.

Cabe insistir, en primer lugar, que una educación científica como la practicada hasta aquí, tanto en la secundaria como en la misma universidad, centrada casi exclusivamente en los 
aspectos conceptuales, es igualmente criticable como preparación de futuros científicos. Ya hemos señalado que esta orientación transmite una visión deformada y empobrecida de la actividad científica, que no solo contribuye a una imagen pública de la ciencia como algo ajeno e inasequible -cuando no directamente rechazable-, sino que está haciendo disminuir drásticamente el interés de los jóvenes por dedicarse a la misma (Matthews, 1991; Solbes y Vilches, 1997).

La gravedad y la extensión de estas deformaciones ha sido puesta de relieve por numerosas investigaciones, que han mostrado las discrepancias entre la visión de la ciencia proporcionada por la epistemología contemporánea y ciertas concepciones y prácticas docentes, ampliamente extendidas, que conciben la actividad científica como un conjunto rígido de etapas a seguir mecánicamente (observación, acumulación de datos,...), resaltando lo que supone tratamiento cuantitativo, control riguroso, etc., pero olvidando -o, incluso, rechazando- todo lo que significa invención, creatividad, duda... (Fernández et al., 2002). Por otra parte, los conocimientos científicos fruto de esta metodología aparecen como "descubrimientos" de genios aislados, encerrados en torres de marfil y ajenos a las necesarias tomas de decisión, ignorándose así tanto el papel del trabajo colectivo, de los intercambios entre equipos, como las complejas relaciones CTS. Todo ello hace que una enseñanza supuestamente dirigida a la formación de científicos, afiance, por acción u omisión (al centrarse únicamente en los aspectos conceptuales y su aplicación operativa), una imagen de la ciencia que, además de falsa, resulta escasamente atractiva, dificultando la génesis de vocaciones científicas (Solbes y Vilches, 1997).

Cabe resaltar, además, que esta enseñanza centrada en los aspectos conceptuales, dificulta, paradójicamente, el aprendizaje conceptual. En efecto, la investigación en didáctica de las ciencias está mostrando que "los estudiantes desarrollan mejor su comprensión conceptual y aprenden más acerca de la naturaleza de la ciencia cuando participan en investigaciones científicas, con tal de que haya suficientes oportunidades y apoyo para la reflexión" (Hodson, 1992). Dicho con otras palabras, lo que la investigación está mostrando es que la comprensión significativa de los conceptos exige superar el reduccionismo conceptual y plantear la enseñanza de las ciencias como una actividad, próxima a la investigación científica, que integre los aspectos conceptuales, procedimentales y axiológicos.

Tras la idea de alfabetización científica no debe verse, pues, una "desviación” o "rebaja” para hacer asequible la ciencia a la generalidad de los ciudadanos, sino una reorientación de la enseñanza absolutamente necesaria también para los futuros científicos; necesaria para 
modificar la imagen deformada de la ciencia hoy socialmente aceptada y luchar contra los movimientos anti-ciencia que se derivan; necesaria incluso, insistimos, para hacer posible una adquisición significativa de los conceptos.

De ninguna forma puede aceptarse, pues, que el habitual reduccionismo conceptual constituya una exigencia de la preparación de futuros científicos, contraponiéndola a las necesidades de la alfabetización científica de los ciudadanos y ciudadanas. La mejor formación científica inicial que puede recibir un futuro científico coincide con la orientación a dar a la alfabetización científica del conjunto de la ciudadanía. Esta convergencia se muestra de una forma todavía más clara cuando se analizan con algún detalle las propuestas de alfabetización científica y tecnológica (Bybee, 1997). La tesis básica de Bybee -coincidente, en lo esencial, con numerosos autores- es que dicha alfabetización exige, precisamente, la inmersión de los estudiantes en una cultura cientifica.

\section{La alfabetización como inmersión en una cultura científica, potenciadora del espíritu crítico}

Nos recuerda Bybee que una de las formas más eficaces de alfabetizarse en una lengua es por inmersión en la cultura de esa lengua. Similarmente, sugiere, cabe suponer que la inmersión en una cultura científica constituya una forma excelente de favorecer la alfabetización científica. Esta tesis, que supone en definitiva aproximar el aprendizaje de las ciencias a una actividad de investigación, ha sido expresada, de una u otra forma, por numerosos autores y aparece como un fruto esencial de la investigación en didáctica de las ciencias.

Estas estrategias están dirigidas, esencialmente, a implicar a los estudiantes en la construcción de conocimientos, aproximando la actividad que realizan a la riqueza de un tratamiento científicotecnológico de problemas. Se propone, en síntesis, plantear el aprendizaje como un trabajo de investigación y de innovación a través del tratamiento de situaciones problemáticas relevantes para la construcción de conocimientos científicos y el logro de innovaciones tecnológicas susceptibles de satisfacer determinadas necesidades. Ello ha de contemplarse como una actividad abierta y creativa, debidamente orientada por el profesor, que se inspira en el trabajo de científicos y tecnólogos, y que debería incluir toda una serie de aspectos como los que enumeramos seguidamente (Gil et al., 1999):

* La discusión del posible interés y relevancia de las situaciones propuestas que dé sentido a su estudio y evite que los alumnos se vean sumergidos en el tratamiento de una situación sin haber 
podido siquiera formarse una primera idea motivadora o contemplado la necesaria toma de decisiones, por parte de la comunidad científica, acerca de la conveniencia o no de dicho trabajo, teniendo en cuenta su posible contribución a la comprensión y transformación del mundo, sus repercusiones sociales y medioambientales, etc.

* El estudio cualitativo, significativo, de las situaciones problemáticas abordadas, que ayude a comprender y acotar dichas situaciones a la luz de los conocimientos disponibles, de los objetivos perseguidos... y a formular preguntas operativas sobre lo que se busca, lo que supone una ocasión para que los estudiantes comiencen a explicitar funcionalmente sus concepciones.

* La invención de conceptos y emisión de hipótesis fundamentadas en los conocimientos disponibles, susceptibles de focalizar y orientar el tratamiento de las situaciones, al tiempo que permiten a los estudiantes utilizar sus 'concepciones alternativas' para hacer predicciones susceptibles de ser sometidas a prueba.

* La elaboración y puesta en práctica de estrategias de resolución, incluyendo, en su caso, el diseño y realización de montajes experimentales para someter a prueba las hipótesis a la luz del cuerpo de conocimientos de que se dispone, lo que exige un trabajo de naturaleza tecnológica para la resolución de los problemas prácticos que suelen plantearse, como, por ejemplo, la disminución de las incertidumbres en las mediciones. Llamamos particularmente la atención sobre el interés de estos diseños y realización de experimentos que exigen (y ayudan a desarrollar) una multiplicidad de habilidades y conocimientos. Se rompe así con los aprendizajes mal llamados 'teóricos' (en realidad simplemente librescos) y se contribuye a mostrar la estrecha vinculación ciencia-tecnología.

* El análisis y comunicación de los resultados, cotejándolos con los obtenidos por otros grupos de estudiantes y asomándose a la evolución conceptual y metodológica experimentada históricamente por la comunidad científica. Ello puede convertirse en ocasión de conflicto cognoscitivo entre distintas concepciones, tomadas todas ellas como hipótesis, y favorecer la 'autorregulación' de los estudiantes, obligando a concebir nuevas conjeturas, o nuevas soluciones técnicas, y a replantear la investigación. Es preciso detenerse aquí en la importancia de la comunicación como substrato de la dimensión colectiva del trabajo científico y tecnológico. Ello supone que los estudiantes se familiaricen con la lectura y confección de memorias científicas y trabajos de divulgación.

* Las recapitulaciones y consideración de posibles perspectivas: conexión de los conocimientos construidos con otros ya conocidos, considerando su contribución a la construcción de cuerpos 
coherentes de conocimientos que van ampliándose y modificándose, con especial atención al establecimiento de puentes entre distintos dominios científicos, porque representan momentos cumbre del desarrollo científico $\mathrm{y}$, en ocasiones, auténticas revoluciones científicas; elaboración y perfeccionamiento de los productos tecnológicos que se buscaban o que son concebidos como resultado de las investigaciones realizadas, lo que contribuye a romper con tratamientos excesivamente escolares y reforzar, así, el interés por la tarea; planteamiento de nuevos problemas... Todo ello se convierte en ocasión de manejo reiterado de los nuevos conocimientos en una variedad de situaciones, contribuyendo a su profundización y resaltando en particular las relaciones Ciencia, Tecnología, Sociedad y Ambiente (CTSA) que enmarcan el desarrollo científico, con atención a las repercusiones de toda índole de los conocimientos científicos y tecnológicos (desde la contribución de la ciencia y la técnica al desarrollo de la humanidad a los graves problemas que hipotecan su futuro), propiciando a este respecto la toma de decisiones, en la forma que ya hemos discutido en el apartado precedente.

Cabe insistir, además, en la necesidad de dirigir todo este tratamiento a mostrar el carácter de cuerpo coherente que tiene toda ciencia, favoreciendo, para ello, las actividades de síntesis (esquemas, memorias, recapitulaciones, mapas conceptuales...) y la elaboración de productos, susceptibles de romper con planteamientos excesivamente escolares, de reforzar el interés por la tarea y de mostrar la estrecha vinculación ciencia-tecnología.

Es conveniente remarcar, así mismo, que las orientaciones precedentes no constituyen un algoritmo que pretenda guiar paso a paso la actividad de los alumnos, sino indicaciones genéricas que llaman la atención sobre aspectos esenciales en la construcción de conocimientos científicos que, a menudo, no son suficientemente tenidos en cuenta en la educación científica. Nos referimos tanto a los aspectos metodológicos como a los axiológicos: relaciones CTSA, toma de decisiones, comunicación de los resultados... El aprendizaje de las ciencias es concebido, así, como un proceso de investigación orientada que permite a los alumnos participar colectivamente en la aventura de enfrentar problemas relevantes y (re)construir los conocimientos cientificos.

Se pretende favorecer así una cierta inmersión en la cultura científica y tecnológica, fundamental para la formación de ciudadanas y ciudadanos críticos que habrán de participar en la toma de decisiones... e igualmente fundamental para que los futuros cientificos logren una mejor apropiación de los conocimientos elaborados por la comunidad científica. 
Puede afirmarse, pues, que la ciencia, cuando no se ve limitada por reduccionismos distorsionantes y empobrecedores, supone una indudable contribución a la formación del espíritu crítico, necesario para no quedar prisioneros de las "evidencias" y de lo que "siempre se ha hecho", y para la toma de decisiones fundamentadas en torno a problemas con serias implicaciones éticas. Resulta necesario y posible que la educación científica recupere esta dimensión.

\section{Conclusión: la cultura científica como fuente de disfrute personal}

Nos hemos referido a las aportaciones de la ciencia al desarrollo histórico de un espíritu crítico liberador y a la resolución de los problemas a los que se enfrenta la humanidad, así como a la posibilidad de una alfabetización científica que extienda a toda la población ambas cualidades.

Pero quisiéramos, para terminar, mencionar una tercera e importante razón para universalizar la educación científica, que va más allá de su utilidad: nos referimos al goce generado por las construcciones científicas que han ampliado nuestra visión del universo, hablándonos de su pasado y de su futuro, ayudándonos a comprender fenómenos que durante milenios espantaron a los seres humanos, contribuyendo a liberarnos de numerosos prejuicios y transmitiéndonos la emoción de apasionantes desafíos.

Para Fensham (2002 b), esta capacidad de la ciencia, como "fuente de placer", para "sorprender y maravillar", señalaría la orientación más conveniente de una educación científica para todos, dejando de lado cualquier ingenua pretensión de preparar a la ciudadanía para participar en la toma de decisiones.

Estamos de acuerdo en la importancia de la educación científica como fuente de placer, pero, como hemos intentado mostrar, la preparación de los ciudadanos y ciudadanas para la toma de decisiones no constituye ninguna ingenua pretensión, sino una necesidad fundamentada. Y, por otra parte, ¿acaso los mayores goces no están asociados a la acción, más allá de la mera contemplación? Poder participar en la superación de algún desafío, ¿no produce un placer superior al de simplemente conocer lo que otros hicieron? Contribuir, como ciudadanas y ciudadanos responsables, a la orientación de nuestro futuro, ¿no es fuente de pasión y de satisfacciones? El disfrute de la cultura científica es un derecho que es preciso promover en toda su plenitud, a través de una inmersión que no se limite a una contemplación externa. Un derecho que es preciso garantizar en beneficio de toda la humanidad. 
Las resistencias a una alfabetización científica de una cierta profundidad, sean cuales sean las razones esgrimidas, constituyen una nueva barricada de un viejo elitismo, que se ha visto obligado a retroceder desde el rechazo inicial incluso a la alfabetización más elemental a la lecto-escritura (Gil y Vilches, 2001). Las razones ahora esgrimidas tienen, a nuestro entender, la misma escasa validez.

Digamos, para terminar, que si bien la historia de la ciencia presenta sombras que no deben ser ignoradas, lo mejor de la misma ha contribuido, como ya señaló Langevin (1926), a los movimientos de liberación de los espíritus y de la extensión de los derechos humanos, que tienen como uno de sus principales objetivos la universalización de todas las adquisiciones culturales valiosas de la humanidad. Y éste debe ser el papel de la educación científica, superando reduccionismos y planteamientos elitistas. Éstas son, en síntesis, las razones por las que seguimos reclamando hoy la alfabetización científica de la ciudadanía.

\section{Referencias bibliográficas}

ATKIN, J.M. y HELMS, J. (1993). Getting serious about priorities in science education. Studies in Science Education, 21, 1-20.

BYBEE, R. W. (1991). Planet Earth in crisis: how should science educators respond? The American Biology Teacher, 53 (3), 146-153.

BYBEE, R. (1997). Towards an Understanding of Scientific Literacy. En Gräber, W. y Bolte, C. (Eds) Scientific Literacy. Kiel: IPN.

CARSON, R. (1980). Primavera Silenciosa, Barcelona: Grijalbo.

COMISIÓN MUNDIAL DEL MEDIO AMBIENTE Y DEL DESARROLLO (1988). Nuestro Futuro Común. Madrid: Alianza.

DEBOER, G.B. (2000). Scientific literacy: another look at its historical and contemporary meanings and its relationship to science education reform. Journal of Research in Science Teaching, 37(6), 582-601.

DECLARACIÓN DE BUDAPEST (1999). Marco general de acción de la declaración de Budapest, http://www.oei.org.co/cts/budapest.dec.htm.

EDWARDS, M., GIL-PÉREZ, D., VILCHES, A., PRAIA, J., VALDÉS, P., VITAL, M.L., CAÑAL, P., DEL CARMEN, L., RUEDA, C. y TRICÁRICO, H. (2001). Una propuesta para la transformación de las percepciones docentes acerca de la situación del mundo. Primeros resultados. Didáctica de las Ciencias Experimentales y Sociales, 15, 37-67. 
FENSHAM, P.J. (2002a). Time to change Drivers for Scientific Literacy. Canadian Journal of Science, Mathematics and Technology Education, 2(1), 9-24.

FENSHAM, P.J. (2002b). De nouveaux guides pour l'alphabétisation scientifique. Canadian Journal of Science, Mathematics and Technology Education, 2(2), 133-149.

FERNÁNDEZ, I., GIL, D., CARRASCOSA, J., CACHAPUZ, A. Y PRAIA, J. (2002). Visiones deformadas de la ciencia transmitidas por la enseñanza. Enseñanza de las Ciencias, $20(3), 477-488$.

FOUREZ, G. (1997). Alfabetización científica y tecnológica. Acerca de las finalidades de la enseñanza de las ciencias. Buenos Aires: Colihue.

GIL, D., CARRASCOSA, J., DUMAS-CARRÉ, A., FURIÓ, C., GALLEGO, R., GENÉ, A., GONZÁleZ, E., GUISASOLA, J., MARTÍNEZ TORREGROSA, J., PESSOA DE CARVAlHO, A. Ma , SAlinAS, J., TRICÁRICO, H. y VALDÉS, P. (1999). ¿Puede hablarse de consenso constructivista en la educación científica? Enseñanza de las ciencias, 17(3), pp. 503-520.

GIL, D. y VILCHES, A. (2001). Una alfabetización científica para el siglo XXI. Obstáculos y propuestas de actuación. Investigación en la Escuela, 43, pp.27-37.

GIL-PÉREZ, D., VILCHES, A., EDWARDS, M., PRAIA, J., MARQUES, L. Y OLIVEIRA, T. (2003). A proposal to enrich teachers' perception of the state of the world. First results. Environmental Education Research, 9(1), 67-90

HICKS, D. y HOLDEN, C. (1995). Exploring The Future A Missing Dimension in Environmental Education”. Environmental Education Research, 1(2), 185-193.

HODSON, D. (1992). In search of a meaningful relationship: an exploration of some issues relating to integration in science and science education. International Journal of Science Education, 14(5), 541-566.

LANGEVIN, P. (1926). La valeur éducative de l'histoire des sciences. Bulletin de la Société Française de Pédagogie, 22, décembre 1926.

MARCO, B. (2000). La alfabetización científica. En Perales, F. Y Cañal, P. (Eds.): Didáctica de las Ciencias Experimentales,141-164. Alcoi: Marfil.

MATTHEWS, M. R. (1991). Un lugar para la historia y la filosofía en la enseñanza de las Ciencias. Comunicación, Lenguaje y Educación, 11-12, 141-155.

NATIONAL RESEARCH COUNCIL, (1996). National Science Education Standards. Washington, D.C.: National Academy Press. 
SÁNCHEZ RON, M. (1994). ¿El conocimiento científico prenda de felicidad? En Nadal J., (Ed), El mundo que viene. Madrid: Alianza.

SHAMOS, M. (1995). The Myth of Scientific Literacy. New Brunswick (NJ): Rutgers University Press.

SOLBES, J. y VILCHES, A. (1997). STS interactions and the teaching of Physics and Chemistry. Science Education, 81(4), 377-386.

VILCHES, A. y GIL, D. (2003). Construyamos un futuro sostenible. Diálogos de supervivencia. Madrid: Cambridge University Press. 